\title{
Bright Field Neuronal Preparation Optimized for Automatic Computerized Reconstruction, a Case with Cerebellar Climbing Fibers
}

\author{
Izumi Sugihara
}

Published online: 2 February 2011

(C) Springer Science+Business Media, LLC 2011

Keywords Climbing fiber-Olivocerebellar projection . Purkinje cell - Axonal reconstruction - Tracer injection · Biotinylated dextran amine $\cdot$ Serial section .

Diaminobenzidine

To make the best use of computerized automatic reconstruction it is crucial to supply optimized neuronal preparations for reconstruction. For bright field preparations a major technique for labeling a specific population of neurons is the injection of tracers, such as dextran conjugated with biotin (biotinylated dextran amine, BDA). BDA-labeled neurons and axons can then be visualized in brain sections using the diaminobenzidine (DAB) reaction, which causes BDA to form a dark precipitate. It is important for such brain sections to be oriented in an appropriate direction so that labeled neurons and axons are localized within individual sections to the greatest extent possible, because automatic reconstruction can be applied only within single brain sections (thickness of 50-100 $\mu \mathrm{m}$ ) at the moment. In this sense, cerebellar climbing fibers, which are organized within a flat longitudinal plane, are among the best suited neuronal elements for automatic reconstruction. For automatic reconstruction, it is also important to separate individual, labeled neurons (or axons) from each other by controlling the amount of tracer. By solving these points the histological preparation can be optimized for automatic computerized reconstruction.

\footnotetext{
I. Sugihara $(\square)$

Department of Systems Neurophysiology,

Tokyo Medical and Dental University Graduate School,

1-5-45 Yushima, Bunkyo-ku,

Tokyo 113-8519, Japan

e-mail: isugihara.phy1@tmd.ac.jp
}

Developers and users of automatic reconstruction software are usually different individuals. Mutual understanding of the technical difficulties and scientific needs on either side is indispensable to optimize collaboration between the two groups. We provided bright-field preparation of climbing fibers labeled with $\mathrm{BDA}^{1,2}$ to the DIADEM challenge. By looking at how the automatic reconstruction software works in the DIADEM conference $^{3,4,5,6,7,8,9}$, I realized that whether the computerized

\footnotetext{
${ }^{1}$ Sugihara, I., Wu, H. -S., \& Shinoda, Y. (1999). Morphology of single olivocerebellar axons labeled with biotinylated dextran amine in the rat. Journal of Comparative Neurology, 414, 131-148.

${ }^{2}$ Sugihara, I., Wu, H. -S., \& Shinoda, Y. (2001). The entire trajectories of single olivocerebellar axons in the cerebellar cortex and their contribution to cerebellar compartmentalization. Journal of Neuroscience, 21, 7715-7723.

${ }^{3}$ Brown, K. M., Barrionuevo, G., Canty, A. J., De Paola, V., Hirsch, J. A., Jefferis, G. S. X. E., et al. (2011). The DIADEM data sets: representative light microscopy images of neuronal morphology to advance automation of digital reconstructions. Neuroinformatics, doi:10.1007/s12021-010-9095-5.

${ }^{4}$ Bas, E., \& Erdogmus, D. (2011). Principal curves as skeletons of tubular objects: Locally characterizing the structures of axons. Neuroinformatics, doi:10.1007/s12021-011-9105-2.

${ }^{5}$ Chothani, P., Mehta, V., \& Stepanyants, A. (2011). Automated tracing of neurites from light microscopy stacks of images. Neuroinformatics, doi:10.1007/s12021-011-9121-2.

${ }^{6}$ Turetken, E., González, G., Blum, C., \& Fua, P. (2011). Automated reconstruction of dendritic and axonal trees by global optimization with geometric priors. Neuroinformatics, doi:10.1007/s12021-011-9122-1.

${ }^{7}$ Zhao, T., Xie, J., Amat, F., Clack, N., Ahammad, P., Peng, H., et al. (2011). Automated reconstruction of neuronal morphology based on local geometrical and global structural models. Neuroinformatics, doi:10.1007/s12021-011-9120-3.

${ }^{8}$ Wang, Y., Narayanaswamy, A., Tsai, C., \& Roysam, B. (2011). A Broadly Applicable 3-D Neuron Tracing Method Based on OpenCurve Snake. Neuroinformatics, doi:10.1007/s12021-011-9110-5.

${ }^{9}$ Narayanaswamy, A., Wang, Y., \& Roysam, B. (2011). 3-D Image Pre-processing Algorithms for Improved Automated Tracing of Neuronal Arbors. Neuroinformatics, doi:10.1007/s12021-011-9116-z.
} 
reconstruction is successful or not depends primarily on the quality of the preparation. In this sense it is important to make preparations that are optimized for the automatic computer reconstruction software. By doing so, it should be possible to eliminate software errors and obtain the best results possible using the software algorithm. Furthermore, knowledge about the extent to which the quality of the labeled material can be controlled may reduce demands on the software, and facilitate further improvements of the software.

Labeling a neuron consists of multiple experimental steps. Systematic and theoretical understanding of the whole procedure is required to tune each step to automatic reconstruction. Therefore, in this article, I will describe some technical issues regarding how a neuron is labeled, and how a preparation can be optimized for automatic reconstruction. The description is partially based on our neuroanatomical experiences in which we labeled, and manually reconstructed, cerebellar climbing fibers and other cerebellar projecting axons ${ }^{1,2,10}$. However, several points that have to be accommodated for automatic reconstruction are emphasized.

\section{Visualizing Neurons for Automatic Reconstruction}

Currently, automatic reconstruction is done in two steps ${ }^{3}$. First, a series of photomicrographs of a labeled neuron are taken every few micrometers of depth ( $\mathrm{Z}$ coordinate) with a digital camera attached to a bright-field microscope. Second, the photomicrographs (graphic files) are processed with software to produce a three-dimensional reconstruction. The shape of the labeled neuron is reconstructed based on the darkness (color) information of every pixel. Therefore, the labeling has to be dark enough ("welllabeled") and the background relatively transparent so that there is good contrast between the two. The labeling also has to represent the original shape of the neuron as exactly as possible. In this perspective, a chromogen, 3,3'-diaminobenzidine tetrahydrochloride (DAB), which is commonly used for bright-field visualization and manual reconstruction of neuronal components, can also be used for automatic reconstruction. Since DAB reacts with peroxidase plus hydrogen peroxide to deposit stable black precipitate, extrinsic peroxidase has to be connected to the target neuron. There are a variety of methods for connecting the extrinsic peroxidase to a specific population of neurons. The method that is most frequently used may be immuno-

\footnotetext{
${ }^{10}$ Sugihara, I., Fujita, H., Na, J., Quy, P. N., Li, B. Y., \& Ikeda, D. (2009). Projection of reconstructed single Purkinje cell axons in relation to the cortical and nuclear aldolase $\mathrm{C}$ compartments of the rat cerebellum. Journal of Comparative Neurology, 512, 282-304.
}

histochemistry, in which a protein expressed in a population of neurons is detected by a specific antibody. In subsequent histological reaction, extrinsic peroxidase is connected to the antibody and utilized to react with $\mathrm{DAB}^{11}$.

A major technique that leads to labeling of a specific population of neurons is injection of an extrinsic substance (a tracer) to that population of neurons. Tracers are required to be resistant to degeneration, transported efficiently through the axon, and to be connected to peroxidase by subsequent histological procedures (except when the tracer is peroxidase itself). There are various ways of making a tracer injection. A tracer can be injected directly inside a single neuron by passing electric current through a glass microelectrode that is used for intracellular recording ${ }^{12}$ or through a patch pipette when doing whole-cell patch clamp recording. Extracellular injection, which is technically much easier than intracellular injection, has been made possible by development of tracers that can be taken up efficiently by neurons. Such tracers include dextran ${ }^{13}$, which is polysaccharide made of glucose molecule synthesized by bacteria, and Phytohemagglutinin-L (PHA-L) ${ }^{14}$, which is extracted from Phaseolus vulgaris (red kidney bean). While PHA-L is visualized by immunostaining using a specific antibody against PHA-L, dextran is used in a form in which it is conjugated to biotin (biotinylated dextran amine, BDA). Biotin $\left(\mathrm{C}_{10} \mathrm{H}_{16} \mathrm{~N}_{2} \mathrm{O}_{3} \mathrm{~S}\right)$ is a small molecule, which specifically connects with avidin, a protein contained in egg-white. By histological procedures that begin with biotin-avidin reaction, peroxidase can be eventually connected to BDA to allow bright-field visualization with DAB. Other groups of tracers include genetically manipulated plasmids of viruses, which induce production of certain proteins in a transfected neuron ${ }^{15}$.

Neurons can also be labeled by using its intrinsic properties without injecting tracers. For example, Golgi

\footnotetext{
${ }^{11}$ Hsu, S. M., Raine, L, \& Fanger, H. (1981). Use of avidin-biotinperoxidase complex $(\mathrm{ABC})$ in immunoperoxidase techniques: a comparison between $\mathrm{ABC}$ and unlabeled antibody (PAP) procedures. Journal of Histochemistry and Cytochemistry, 29, 577-580.

${ }^{12}$ McCrea, R. A., Bishop, G. A., \& Kitai, S. T. (1976). Intracellular staining of Purkinje cells and their axons with horseradish peroxidase. Brain Research, 118, 132-136.

${ }^{13}$ Veenman, C. L., Reiner, A., \& Honig, M. G. (1992). Biotinylated dextran amine as an anterograde tracer for single- and double-labeling studies. Journal of Neuroscience Methods, 41, 239-254.

${ }^{14}$ Gerfen, C. R., \& Sawchenko, P. E. (1984). An anterograde neuroanatomical tracing method that shows the detailed morphology of neurons, their axons and terminals: immunohistochemical localization of an axonally transported plant lectin, Phaseolus vulgaris leucoagglutinin (PHA-L). Brain Research, 290, 219-238.

${ }^{15}$ Matsuda, W., Furuta, T., Nakamura, K. C., Hioki, H., Fujiyama, F., Arai, R., et al. (2009). Single nigrostriatal dopaminergic neurons form widely spread and highly dense axonal arborizations in the neostriatum. Journal of Neuroscience, 29, 444-453.
} 
staining labels neurons randomly by deposition of metal particles in brain tissue soaked in heavy-metal salt solution. Immunostaining can label a population of neurons that specifically express an antigen molecule. For example, a population of climbing fibers are labeled by immunostaining of corticotrophin-releasing factor ${ }^{16}$.

Of the above techniques, we have been using BDA to label climbing fibers (Fig. 1a). Although BDA labeling seems the most efficient for obtaining preparations appropriate for axonal reconstruction, it is a difficult technique to master. It may take months for one to acquire the technique since conditions to make well-labeled preparation are not always easy to obtain. Therefore, I describe some basic principles of the labeling here. Often, labeling may not be dark enough, may be fragmentary, or may miss fine collaterals, if the axon is not uniformly or completely filled ("weakly-labeled", asterisks, Fig. 1c). On rare occasions when the axon is filled fully, labeling may be so strong as to thicken the appearance of axons. This may distort the fine details of axons since nearby collaterals may appear to merge with each other (arrowhead, Fig. 1c). It is not difficult to distinguish well-labeled axons from weaklylabeled axons or strongly-labeled axons because the labeling intensities are clearly different (Fig. 1c). Reconstruction can be frustrating or impossible with weakly-labeled axons since continuity of axons may be difficult to trace even with very careful observation. Strong labeling may not be a problem unless collaterals of an axon run in close proximity to each other as occurs with climbing fibers, in which multiple thin collaterals surround the proximal Purkinje cell dendrite like an ivy vine ${ }^{1,17}$ (Fig. 1b). Thus, observation under a high magnification oil-immersion objective lens with fine $Z$ coordinate displacement may be required to reconstruct details even for optimally labeled climbing fibers. Indeed, to distinguish separate collaterals that run in close proximity was the most difficult task for the reconstruction software ${ }^{4-9}$ in the climbing fiber assignment in the DIADEM challenge.

These technical issues can be partially solved by appropriate care during the experiment; better control of tracer injection amount and better histological procedures, may increase the possibility that one or more neurons (or axons) are well labeled. However, still, despite considerable experience, typically less than half of the labeled neurons (or axons) are well-labeled in our experiments. It is important that only well-labeled neurons (or axons) should be reconstructed, whether by manual or automatic means.

\footnotetext{
$\overline{{ }^{16} \text { Cummings, }}$ S., Sharp, B., \& Elde, R. (1988). Corticotropinreleasing factor in cerebellar afferent systems: a combined immunohistochemistry and retrograde transport study. Journal of Neuroscience, 8, 543-554.

${ }^{17}$ Ramón y Cajal, S. (1911). Histologie du Système Nerveux de l'Homme et des Vertébrés, Vol. II. Paris: Maloine.
}

Thus, to increase the number of reconstructed samples, it is necessary to increase the number of injection experiments, rather than to try to reconstruct non-well-labeled neurons (or axons) from a limited number of experiments.

Besides climbing fibers, BDA labeling can visualize the morphological details of other axons clearly, as the following examples of labeled axons with widely differing morphologies illustrate. Collaterals of climbing fiber axons in the cerebellar nucleus, which are very thin, and have small, sparsely distributed swellings (Fig. 1e). A mossy fiber axon in the cerebellar cortex with its large irregular terminals, termed "rosettes" (Fig. 1f). A Purkinje cell axon, which has large, sphere-shaped and clustering terminals (Fig. 1g). BDA (and PHA-L) labeling has been used in thalamocortical $^{18}$ and other projections, which can be analyzed at the level of single axons ${ }^{19}$.

\section{Sectioning Brain Tissue for Automatic Reconstruction}

The DAB reaction for visualization (above) involves incubating the tissue in solutions of histological reagents. A limit on the thickness of histological sections is imposed by the need for reagents to spread into the depths of the tissue during incubation. Sections usually can be at most 50-100 $\mu \mathrm{m}$. Automatic reconstruction software now works with a single histological section; it is not capable of reconstructing from serial sections at the moment. Thus, a significant portion of the neuron (or neuronal process) must be located within a single section for automatic reconstruction to work. Therefore, neurons that can have a scientifically interesting part contained within a thickness of 50$100 \mu \mathrm{m}$ are candidates for automatic reconstruction. There are many candidates that could fulfill this condition. However, the direction of brain sectioning has to be carefully chosen to increase the probability that all the parts of interest of the neuron lie within a single section.

Of the neuronal circuitry in the vertebrate cerebellum, a climbing fiber (Fig. 1a) matches with this condition most well. A climbing fiber is the terminal portion of the axon of an inferior olive neuron, and innervates a single Purkinje cell in the cerebellar cortex. A single climbing fiber gives rise to an abundant axonal arborization that generates about 250 swellings or varicosities that form synapses onto the proximal dendrites of the Purkinje cell ${ }^{1}$. A Purkinje cell has

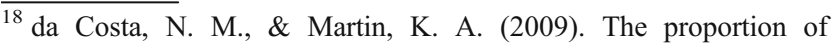
synapses formed by the axons of the lateral geniculate nucleus in layer 4 of area 17 of the cat. Journal of Comparative Neurology, 516, 264 276.

${ }^{19}$ Rockland, K. S. (2002). Visual cortical organization at the single axon level: a beginning. Neuroscience Research, 42, 155-166. Review.
} 

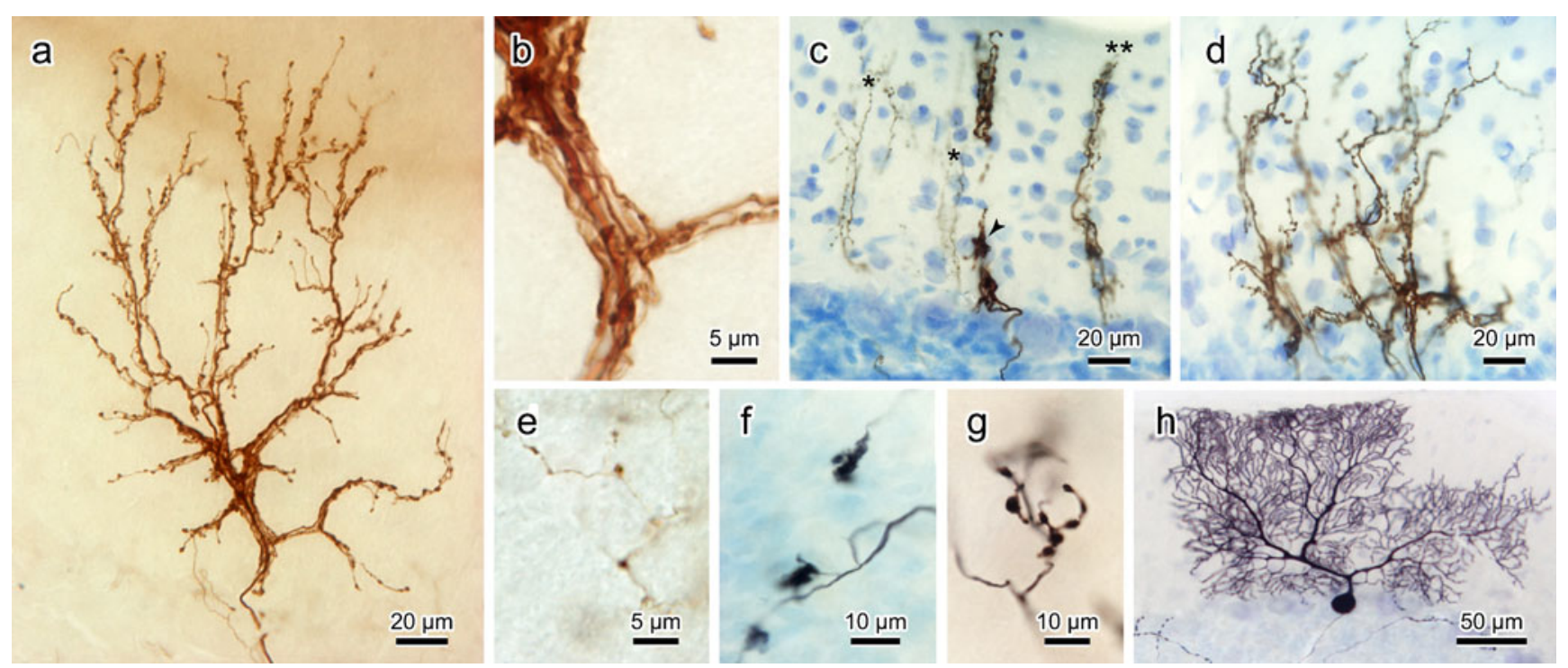

Fig. 1 Photomicrographs of labeled climbing fibers and other axons of adult rat cerebellum. a, A well-labeled climbing fiber that is arranged in parallel with the section. Montage of five photographs with slightly different $\mathbf{z}$-axis focusing. $\mathbf{b}$, The proximal portion of the climbing fiber shown in A. High magnification with oil-immersion lens shows multiple fine collaterals. c, Climbing fibers that are arranged perpendicularly to the section. One climbing fiber was welllabeled $(* *)$, two climbing fibers are faintly labeled $(*)$, and another

climbing fiber was strongly-labeled (arrowhead). d, Five climbing fibers that are well-labeled and arranged obliquely to the section. It would be difficult to separate them in automatic reconstruction. e, A collateral of a climbing fiber axon in the cerebellar nucleus. f, Mossy fibers that originate from the gracile nucleus. g, Terminals of a Purkinje cell axon in the cerebellar nucleus. h, A single Purkinje cell that is arranged in parallel to the section. $\mathrm{h}$ is after Sugihara et al. $(2009)^{9}$. Sections were counterstained with thionine in $\mathrm{c}, \mathrm{d}, \mathrm{f}$ and $\mathrm{h}$

a large dendritic arbor, which extends more than $100 \mu \mathrm{m}$ in two directions within a flat longitudinally oriented plane (Fig. 1h). In contrast, the arbor extends for a thickness of only about $8 \mu \mathrm{m}$ in the direction perpendicular to this plane. Accordingly, the climbing fiber that innervates the proximal dendritic arbor of a Purkinje cell is also localized within a similarly thin rectangularly-shaped region (Fig. 1a, c). In rodents, the longitudinal plane is parallel to the midsagittal section in the median and paramedian areas (vermis) of the cerebellum, but is tilted up to about $60^{\circ}$ in the lateral areas (hemisphere). If the direction of brain sectioning is carefully adjusted to match the plane of the Purkinje cell dendritic arbor, it is possible for an entire climbing fiber to be contained within a single brain section of 50-100 $\mu \mathrm{m}$. In longitudinal (sagittal) sections of the cerebellum, climbing fibers in the central vermis are arranged in parallel with the section (Fig. 1a). However, climbing fibers in other areas of the cerebellum are mostly arranged obliquely to the section (Fig. 1d), and when climbing fibers are arranged perpendicular to the section, they look like a thin straight ladder (Fig. 1c). Thus, all parts of a climbing fiber cannot stay within a single section of $\sim 50 \mu \mathrm{m}$ unless the climbing fiber is oriented essentially in parallel to the section.

One of the merits of automatic reconstruction is that mathematical parameters, such as branch number, branch length, swelling number and so on, are obtained once the reconstruction is completed. Such parameters allow one to compare climbing fiber morphology between different

species, different development stages ${ }^{20}$, different animal models ${ }^{21}$ and different genetic backgrounds ${ }^{22}$. A climbing fiber shows significant change in its morphology during development, which has been partially reported by manual reconstruction (Fig. 2a-d). However, these morphological changes have not been quantified sufficiently. Automatic reconstruction may be capable of eliminating this deficit.

\section{Controlling the Injection Amount of the Tracer for Automatic Reconstruction}

To prepare materials optimized for automatic reconstruction with extracellular tracer injection, controlling the injection amount of the tracer is important. Concerning the density of labeled neuronal/axonal population, the number of labeled

\footnotetext{
${ }^{20}$ Sugihara, I. (2005). Microzonal projection and climbing fiber remodeling in single olivocerebellar axons of newborn rats at postnatal days 4-7. Journal of Comparative Neurology, 487, 93-106. ${ }^{21}$ Sugihara, I., Bailly, Y., \& Mariani, J. (2000). Olivocerebellar climbing fibers in the granuloprival cerebellum: morphological study of individual axonal projections in the X-irradiated rat. Journal of Neuroscience, 20, 3745-3760.

${ }^{22}$ Ichikawa, R., Miyazaki, T., Kano, M., Hashikawa, T., Tatsumi, H., Sakimura, K., et al. (2002). Distal extension of climbing fiber territory and multiple innervation caused by aberrant wiring to adjacent spiny branchlets in cerebellar Purkinje cells lacking glutamate receptor delta 2. Journal of Neuroscience, 22, 8487-8503.
} 
Fig. 2 Manually reconstructed immature climbing fibers at postnatal days 4-7 (a-c) and at adult (d). Green circles indicate Purkinje cells. After Sugihara, $(2005)^{20}$
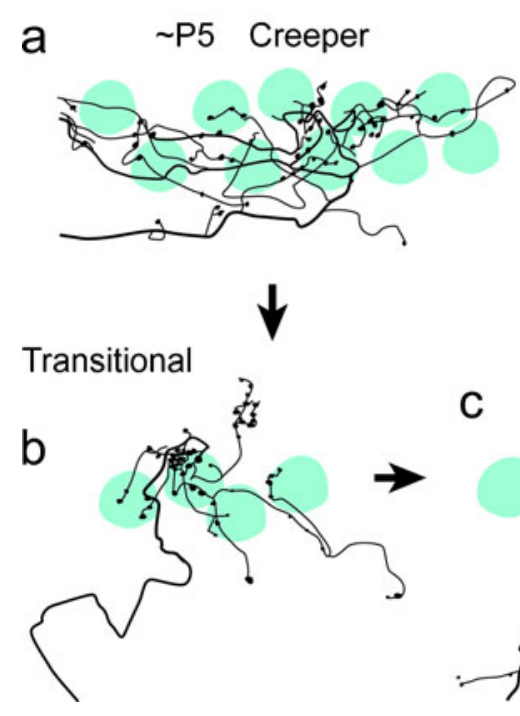

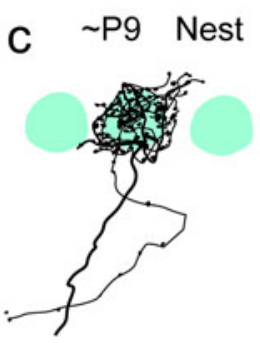

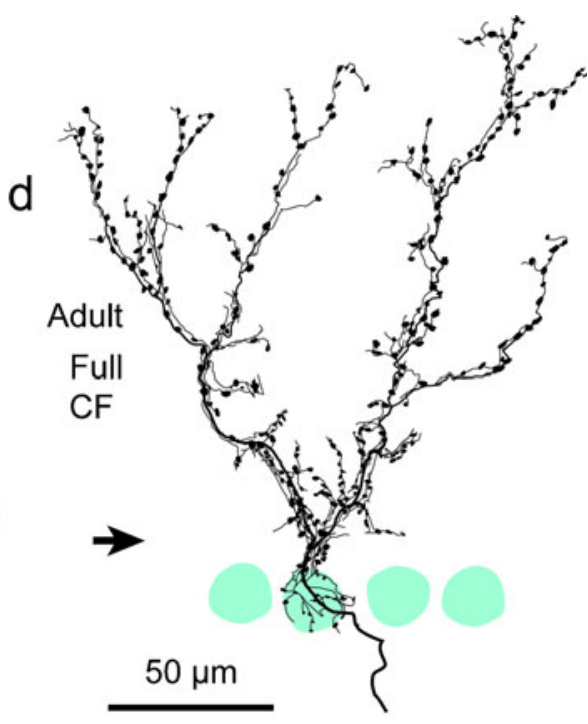

neurons is totally dependent on how the BDA injection is performed. In automatic reconstruction, it is crucial that a single axon/neuron be well separated from other axons/ neurons, because software cannot use cues that human perception uses to distinguish separate axons or neurons when they cross each other at slightly different depths ( $Z$ coordinate) in a section; for example, five adjacent climbing fibers in Fig. 1d would be difficult to be separated. Therefore, it is preferable that there is no overlap of multiple axons/neurons but only a single labeled axon in a given X-Y coordinate. This problem is usually solved by making a small injection so that only a small number of neurons/axons are labeled. Therefore, I will describe this point further for BDA, which is an extracellular tracer that has been most often used for anterograde axonal tracing by many laboratories, including ours.

BDA (D-1956, molecular weight 10,000 or D-7135, molecular weight 3,000; Molecular Probes, Eugene, OR) is dissolved in saline $(10 \%)$ and subdivided into $1.5 \mu \mathrm{l}$ aliquots, which are kept frozen $\left(-80^{\circ}\right)$ in small sample tubes until they are used. Since $10 \%$ BDA solution is already very thick, almost too thick to handle easily, we have not tried higher concentrations. A glass micropipette is formed from a thin glass capillary (outer diameter: $1.5 \mathrm{~mm}$ ) with or without an internal glass filament. The tip of the micropipette is broken to $5 \mu \mathrm{m}$ by hitting with a metal bar under a microscope. Then, BDA solution is poured to the inside of the injection pipette from the back by using thin polyethylene tubing. A thin stainless steel wire is then inserted into the BDA solution in the micropipette to allow electrical recording of neuronal activity. Electrical recording helps in locating the brain area where the injection is to be made. BDA can be injected by electric current or by pressure. We typically use pressure injection with an electronic valve device (Picopump PV820, WPI, Sarasota, FL), which is connected to the injection pipette via polyethelene tubing with the junction between the tubing and pipette sealed with dental wax. The injection amount is calibrated by measuring the approximate diameter of the drop which is pushed out by applying pressure. It takes 20-200 $\mathrm{ms}$ to push $4-40 \mathrm{nl}$ of $10 \%$ BDA solution with a micropipette tip diameter of $\sim 5 \mu \mathrm{m}$ and with an air pressure of $5.0 \mathrm{~atm}(=0.51 \mathrm{MPa}=$ $\left.5.2 \mathrm{kgf} / \mathrm{cm}^{2}=73 \mathrm{psi}\right)$. These parameters are suited to obtain well-labeled axons (also see below). We use a home-made fine measure $(0.1 \mathrm{~mm}$ scale $)$ to calibrate the drop. Following this calibration, the injection pipette is immediately inserted to the brain, and positioned in the target structure under guidance of stereotaxic coordinates and the electrical signals are recorded from the pipette. Once the target location is identified an injection is made. Electrical recording also helps in monitoring changes in neuronal activity that may be induced by an injection. A temporary inactivation of neuronal activity is usually noted as a sign of a successful injection ${ }^{10}$. After a survival period during which BDA is transported throughout the neurons ( 7 days for the rat inferior olive neurons), the animal is perfused and fixed.

Controlling tracer injection amount is important in labeling cerebellar climbing fibers. Although a single olivocerebellar axon gives rise to about seven climbing fibers, the climbing fibers originating from a single olivocerebellar axon usually project to separate areas. Thus, nearby climbing fibers in the cerebellar cortex originate from different (but adjacent) inferior olive neurons. Therefore, if fewer inferior olive neurons are labeled, the probability that neighboring climbing fibers are labeled in the cerebellar cortex becomes smaller. If multiple 
labeled climbing fibers have the same position in terms of their $\mathrm{X}$ and $\mathrm{Y}$ coordinates in a histological section (Fig. 1d), it becomes very difficult to distinguish them with automatic reconstruction software, even if they are located at different depths (different $\mathrm{Z}$ coordinates).

Practically, a small tracer injection in the inferior olive that spreads to a diameter of $\sim 0.2 \mathrm{~mm}$ (volume $\sim 4 \mathrm{nl}$ ) usually labels less than 10 olivocerebellar axons. Such a preparation is appropriate for manual reconstruction of the entire trajectories of these axons. It may also be used for automatic reconstruction, since the labeled climbing fibers are mostly separated from each other. However, a larger injection that spread up to a diameter of $\sim 0.4 \mathrm{~mm}$ (volume $\sim 33 \mathrm{nl}$ ) would also be suitable for automatic reconstruction, since automatic reconstruction would handle larger number of climbing fibers. With this density of labeling, majority of labeled climbing fibers would be still separated from each other. Such an injection can label some tens of olivocerebellar axons or a couple of hundred of climbing fibers in multiple lobules which are topographically related to the injection site in the olive.

\section{Technical Extension Proposed for Automatic Reconstruction}

Since automatic reconstruction of neuronal morphology is a challenging and promising field, I would like to propose some ideas and goals for improving automatic reconstruction methods. Automatic reconstruction from neighboring sections would be useful. The dendritic arbor of a single neuron often spreads wider than $100 \mu \mathrm{m}$, which means it is often divided into multiple consecutive histological sections. Therefore, if automatic reconstruction can be done for neurons and/or axonal terminals that occupy a couple of consecutive sections, the method can be applied to much larger variety of materials. Reconstruction from neighboring sections could then be extended to reconstruction of long axons from many serial sections. To accomplish this goal, the automatic reconstruction system would have to mimic how we manually reconstruct from serial sections. The essential thing is to look for the same axon in the next section. The positional relationship between the identified axon and nearby marker objects such as major structures of the brain and other labeled axons is the key to identifying the same axon in the next section. Thus, the system has to move the slide in $\mathrm{X}$ and $\mathrm{Y}$ direction automatically to search for the same position in the next section. Automatic exchanging of the objective lens, adjustment of $\mathrm{Z}$ axis coordinate, photographing and analysis would all be required.

\section{Conclusion}

To make the best use of computerized automatic reconstruction it is crucial to supply optimized neuronal preparations. Bright-field labeling of neurons consists of multiple experimental steps. In this article, I briefly described these steps and explained how each step can be tuned to prepare materials optimized for automatic computerized reconstruction. A climbing fiber, with which we have been long involved, is a good material to use for automatic reconstruction, because it has a large special terminal arbor that is localized within a thin solid rectangular region. For optimizing the automatic reconstruction, a particularly important experimental parameter is the amount of tracer injected, because it is critical to label appropriate density of neuronal elements (climbing fibers in the example used here), which remain separated from each other.

Acknowledgements Preparation of this article was supported by a Grant-in-Aid for Scientific Research from the Japan Society for the Promotion of Science (20300137). The author thanks Drs. E. J. Lang and $\mathrm{H}$. Fujita for reading and providing valuable comments. 